\title{
Novel plant protein blends for the design of functional foods for older adults to promote healthy ageing
}

\author{
E.T. Olaniyan ${ }^{1}$, F. O’Halloran ${ }^{1}$ and A.L. McCarthy ${ }^{1}$ \\ ${ }^{1}$ Biological Sciences Department, Cork Institute of Technology, Bishopstown, Cork, Ireland
}

Due to anabolic resistance and reduced muscle mass preservation, older adults have increased protein requirements ${ }^{(1)}$. A potential strategy to increase protein in older adults is through developing functional protein snack foods. While animal proteins have a superior amino acid (AA) profile compared to plant proteins, blending plant proteins can improve their amino acid profile and potentially increase their biological activity ${ }^{(2)}$.

The objective of this study was to formulate plant protein blends using commercially available protein isolates (rice, pea, and pumpkin), and investigate their free amino acid profiles (FAA) and antioxidant properties. The blends initially underwent enzyme hydrolysis, then the FAA were quantified using acid hydrolysis and LC-MSMS, while the antioxidant properties were investigated using non-cellular antioxidant assays: Total Phenolic Content (TPC), Ferric Reducing Antioxidant Power (FRAP).

Of the 13 blends formulated, the FAA results showed that blends with a higher ratio of rice had a more favourable AA profile overall, while blends with a higher ratio of pumpkin had the least favourable AA profile. In this experiment, blends with high amounts of branched-chain amino acids (BCAAs) and essential amino acids are regarded as having a favourable AA profile. Blends with a higher ratio of pea had a higher leucine content relative to the other blends, with the highest leucine content recorded as $175 \mathrm{mg} /$ $100 \mathrm{~mL}$ hydrolysate. TPC and FRAP antioxidant assay results agree for the majority of blends, with blends that had a higher ratio of rice protein showing higher TPC activity $(70-87 \mathrm{mg}$ GAE $/ 100 \mathrm{~mL})$, and blends with a higher ratio of pumpkin showing higher FRAP activity $(58 \mathrm{umol} / \mathrm{L}-64 \mathrm{umol} / \mathrm{L})$. Although there were no significant differences $(\mathrm{p}<0.05)$ in the antioxidant properties, all the blends showed high TPC activity. The blends showed high levels of FRAP activity, with the exception of one blend which had the lowest FRAP value of 38 umol/L.

The results of this study suggest that blends with higher amounts of rice and pea protein may be more beneficial in achieving optimal postprandial AA bioavailability. Future studies should investigate if the FAA profiles and antioxidant properties would be retained when the blends are in a food matrix.

\section{Acknowledgements}

This research was supported by a RÍSAM PhD scholarship to Eunice Ti-Oluwani Olaniyan from Cork Institute of Technology.

\section{References}

1. Witard O, Wardle S, Macnaughton L, et al. (2016) Nutrients 8, 181

2. Olaniyan ET, O’Halloran F \& McCarthy A (2020) Nutr Res Rev 1-11 